\title{
Hepatic Angiosarcoma, going but not gone. Lessons from a single centre experience
}

\author{
French $\mathrm{J}^{*}$, Gow $\mathrm{P}^{1}$, Strickland $\mathrm{AH}^{2}$, Testro $\mathrm{A}^{1}$, Angus $\mathrm{P}^{1}$, Goodwin $\mathrm{M}^{3}$ and Crowley $\mathrm{P}^{4}$ \\ ${ }^{1}$ Department of Gastroenterology \& Liver Transplant, Austin Health, Victoria. \\ ${ }^{2}$ Department of Medical Oncology, Monash Cancer Centre, Victoria \\ ${ }^{3}$ Department of Radiology, Austin Health, Victoria \\ ${ }^{4}$ Histolab pathology services, University of Melbourne
}

*Corresponding author: Janine French, Department of Gastroenterology and Hepatology, Austin Hospital, 145 Studley Road, Heidelberg, Australia, Tel: +61 (0) 39496 5353; Fax: +61 (0) 39496 3487; E-mail: janine. french@austin.org.au

Citation: French J, Gow P, Strickland AH, Testro A, Angus P, et al. (2014) Hepatic Angiosarcoma, going but not gone. Lessons from a single centre experience. J Case Rep Stud 2(1): 102. doi: 10.15744/2348-9820.1.402

Received Date: January 12, 2014 Accepted Date: February 20, 2014 Published Date: February 24, 2014

\begin{abstract}
Hepatic angiosarcoma is a rare tumour that is often difficult to diagnose. Historically, most cases of hepatic angiosarcoma were seen in the setting of industrial epidemics caused by exposure of workers to toxins such as vinyl chloride. Cases associated with recognised exposure to carcinogens have fortunately been extremely rare for the last three or more decades. However, the tumour has by no means disappeared in the Australian community. In this case series, we describe three cases of hepatic angiosarcoma that were seen at our institution since 2002. The first case presented with cholestatic liver function tests and was found to have angiosarcoma on liver biopsy. In the second case, the patient was admitted for decompensated liver disease on a background of presumed hepatitis B cirrhosis. The diagnosis of hepatic angiosarcoma was made only at autopsy after the patient died from multi-organ failure. The third case presented with ascites and the diagnosis of disseminated angiosarcoma was again made at autopsy following a negative ante-mortem liver biopsy. Keywords: Angiosarcoma; Liver; Autopsy; Vinyl chloride; Budd Chiari
\end{abstract}

\section{Introduction}

Hepatic angiosarcoma is a very rare disease, accounting for only $2 \%$ of primary liver malignancies [1]. Most patients with this disease only present after they have developed symptoms from extensive invasion of the liver parenchyma.

Hepatic angiosarcoma can occur sporadically, or be associated with certain classical risk factors such as exposure to vinyl chloride, arsenic, thorotrast or other toxins [2]. The outcome of patients who develop hepatic angiosarcoma is poor, attributable to its rapid progression, late presentation, high recurrence rate in cases resected surgically, and resistance to traditional chemotherapy and radiotherapy. The median survival is only 6 months [3].

\section{Case 1}

A 37 year old female on the oral contraceptive pill presented with a two month history of abdominal pain and cholestatic liver function tests (Bilirubin 22 micromol/L ALP 261 units/L, GGT 110 units/L.) Initial abdominal ultrasound showed hepatomegaly and a subsequent CT showed a grossly abnormal vascular pattern in an enlarged liver. The working diagnosis was initially BuddChiari syndrome. The patient subsequently had a normal doppler of her hepatic veins and normal hepatic venography.

CT angiography confirmed patent hepatic veins with an abnormal small vessel pattern thought to be a result of infiltration of the liver parenchyma by tumour. A percutaneous needle core biopsy of the liver was suspicious for angiosarcoma. Subsequent laparoscopic wedge biopsies confirmed the diagnosis, showing a classical pattern of dilated sinusoids lined by proliferating malignant cells growing along preformed liver cell plates in a scaffolding pattern, as well as areas composed of spindle cells. The endothelial nature of these cells was confirmed with immunohistochemistry, which showed staining for CD34, CD31 and factor VIII. The patient was treated with seven cycles of single agent adriamycin with reasonable disease control. When there was 
evidence of disease progression she was switched to thalidomide but had no apparent response and deteriorated rapidly. She died within seven months of the diagnosis.

\section{Case 2}

A 60 year old man presented in the early 1980's with bleeding from oesophageal varices. This was initially managed with a lienorenal and subsequently mesocaval shunt. He was Hepatitis B surface antigen positive and a liver biopsy performed at the time confirmed he had cirrhosis.

In 2002 the patient was admitted to our institution with deteriorating liver function tests. On admission he was icteric and mildly encephalopathic. Coagulation studies were consistent with disseminated intravascular coagulation (INR 6, APTT >240, TCT >60). Liver function tests revealed elevated bilirubin (100 micromol/L) and hypoalbuminaemia (23 g/L).

His initial CT revealed abnormal perfusion of the liver and several arterially enhancing regions in the right lobe with no portal venous phase washout. Whilst the liver lesions were still being investigated the patient developed type 1 hepatorenal syndrome and subsequently died secondary to a massive GI bleed.

Autopsy revealed a liver diffusely replaced by peliosis-like spaces containing blood (Figure 1). Histological examination of the postmortem liver revealed a classical pattern of angiosarcoma extensively replacing the normal liver (Figures $2 \& 3$ ). In addition there were numerous scarred portal veins in keeping with non-cirrhotic portal hypertension (Figure 4).

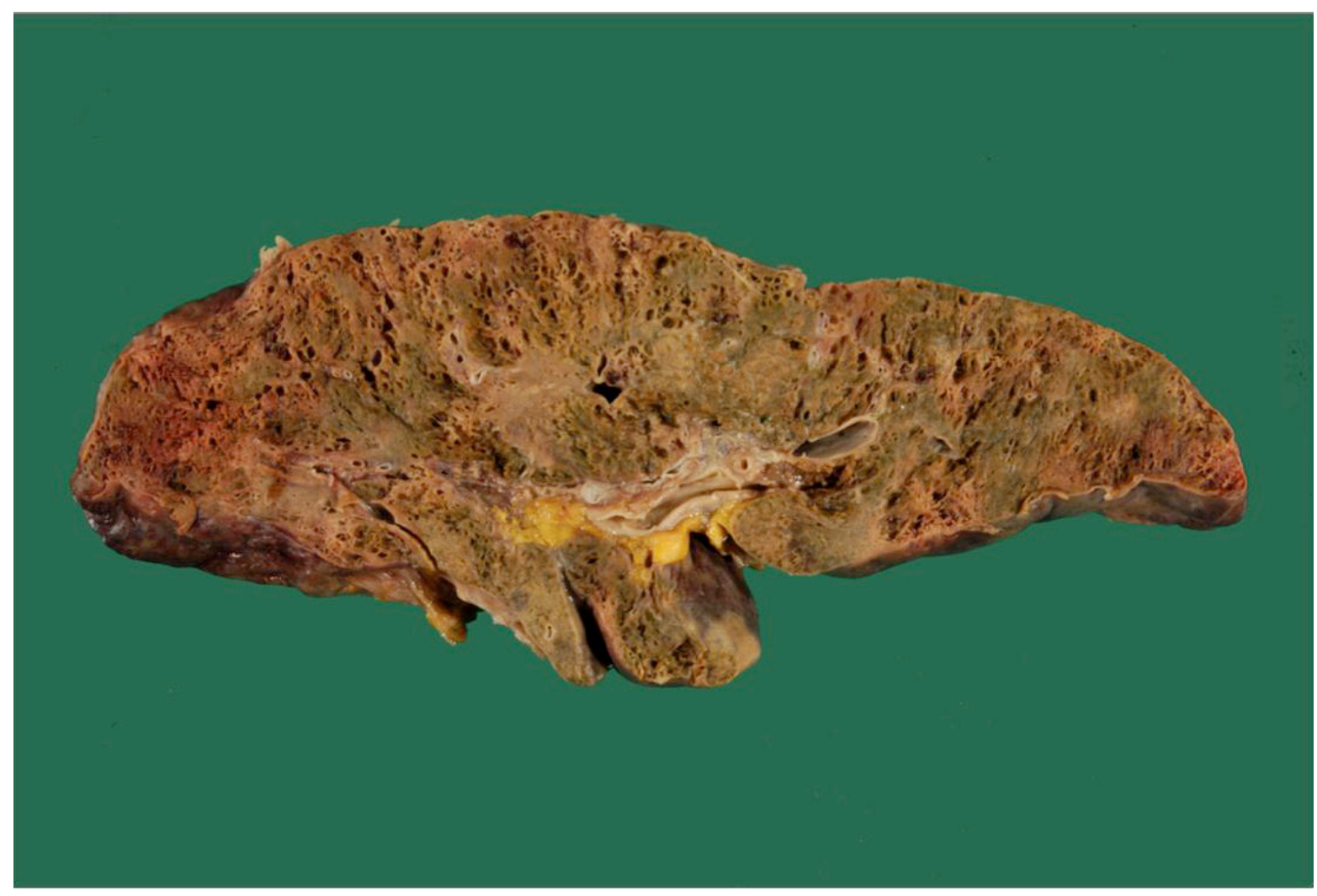

Figure 1: Autopsy liver. The hepatic parenchyma has been replaced by numerous "pelioisis-like" spaces which contained blood 


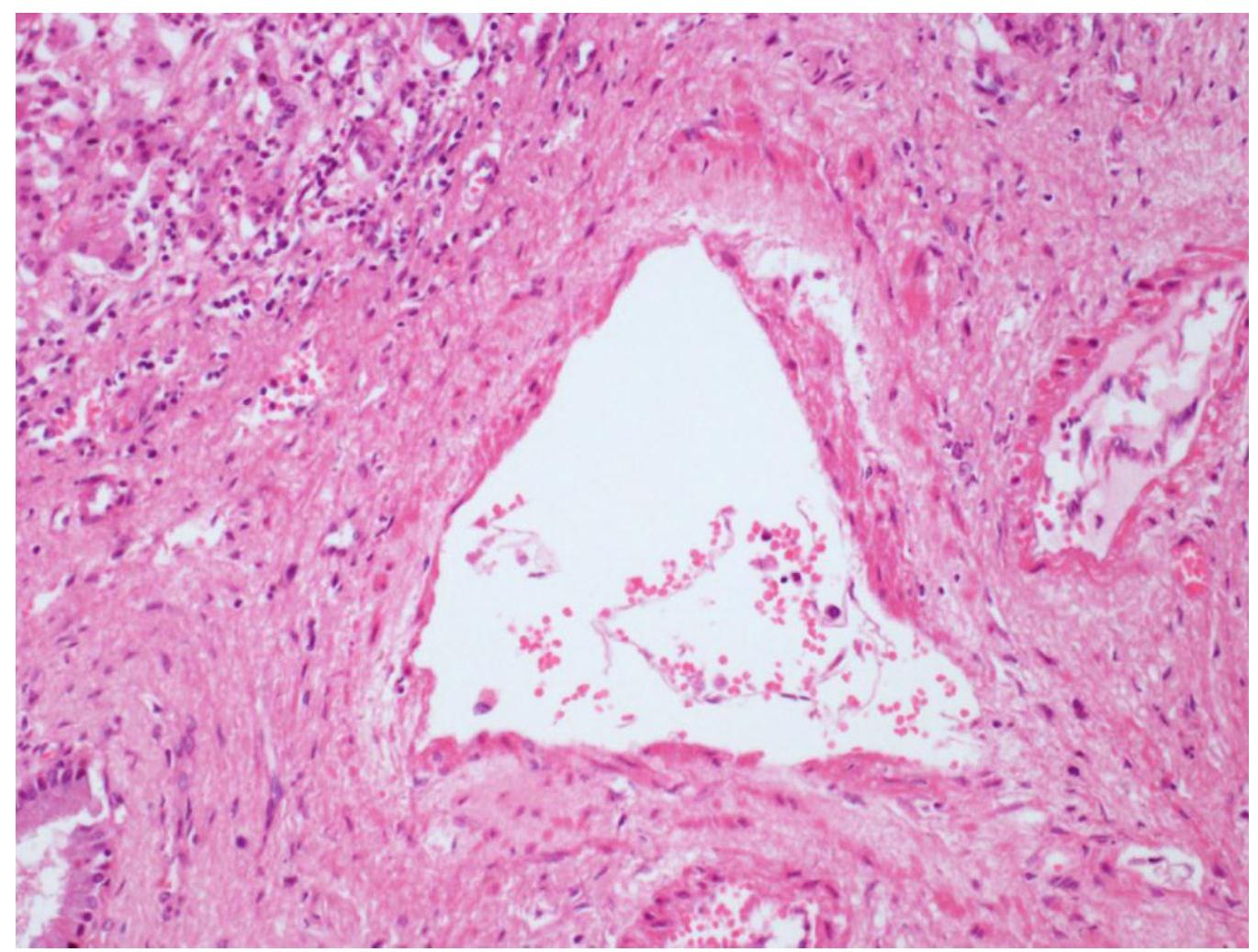

Figure 2: H\&E Stain, autopsy liver. A scarred portal vein consistent with hepatoportal sclerosis (non-cirrhotic portal hypertension)

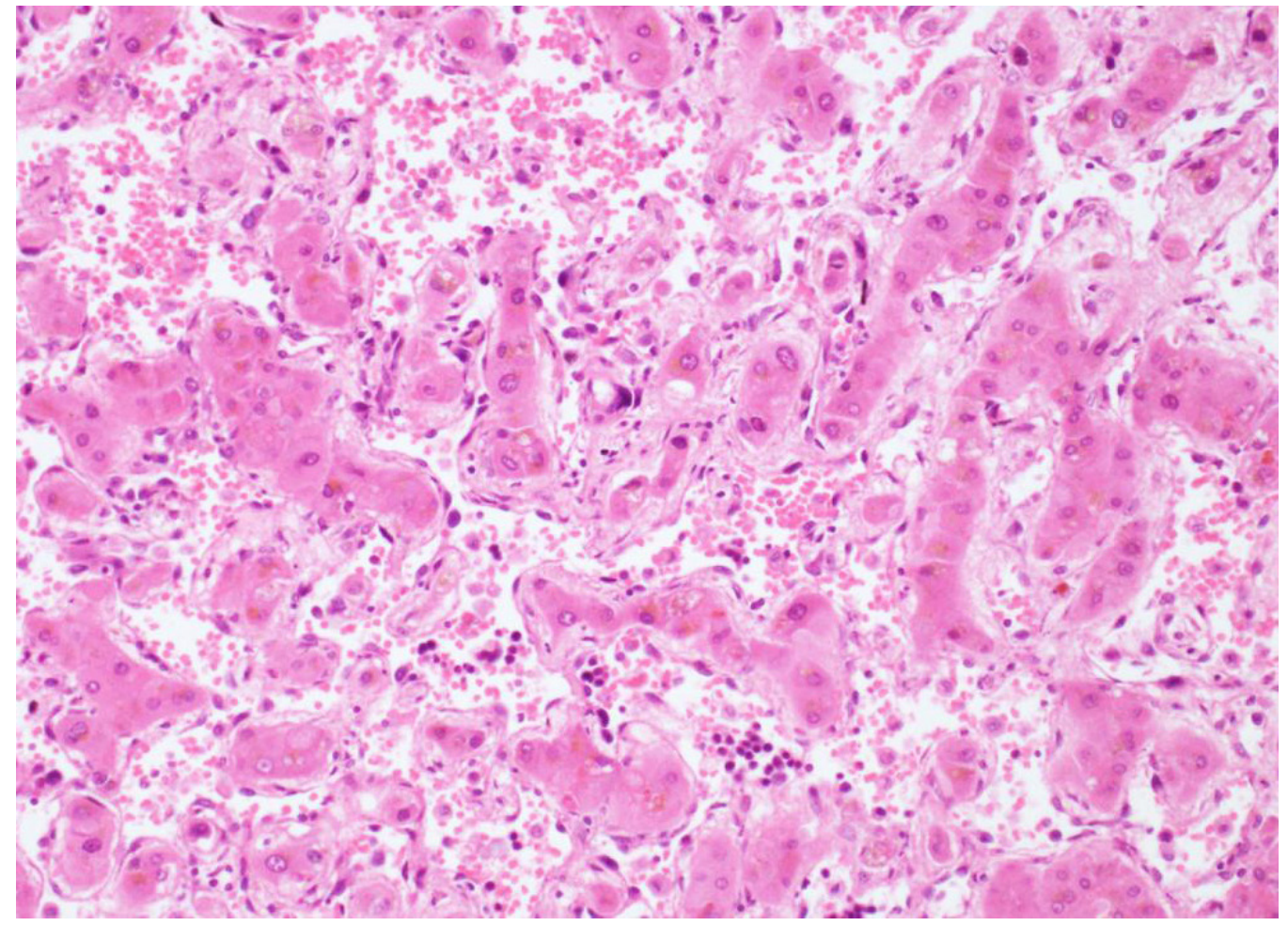

Figure 3: H\&E Stain, autopsy liver. Angiosarcoma. 


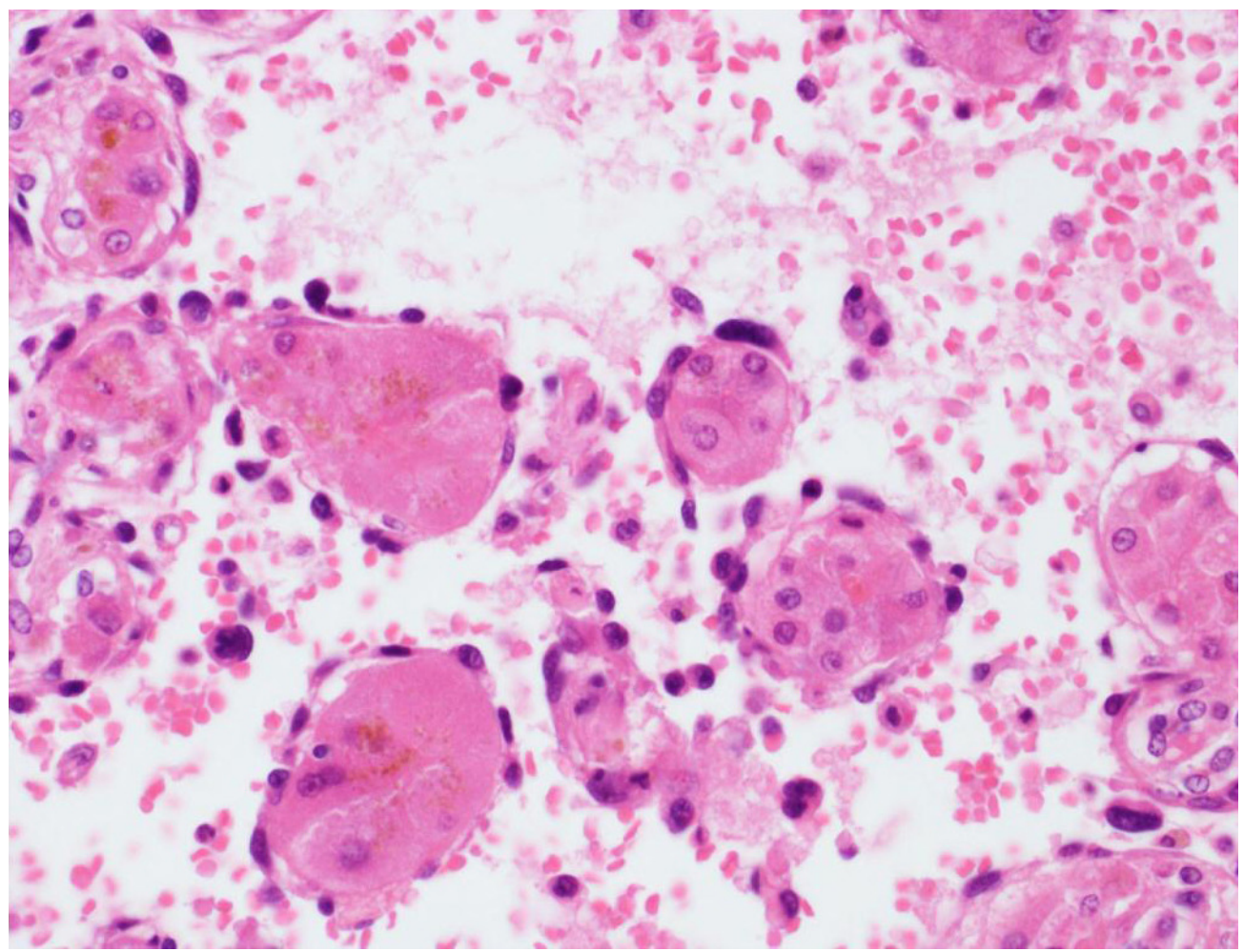

Figure 4: H\&E Stain, autopsy liver. The tumour cells line dilated sinusoids, using thickened liver cell plates as a "scaffold"

Review of the archival liver biopsy showed changes more in keeping with non-cirrhotic portal hypertension (hepatoportal sclerosis) than cirrhosis. There was no evidence of chronic hepatitis. The paraffin block was received for immunohistochemistry. There was no hepatocellular staining for antibodies to Hepatitis B surface or core antigen.

On further questioning of his family post mortem, it was discovered that the patient had known exposure to vinyl chloride whilst working as an engineer forty years previously. Occupational exposure to vinyl chloride is a recognized cause of hepatic angiosarcoma and was responsible for epidemics of this tumour peaking in the west in the 1970s. Non-cirrhotic portal hypertension was also described as a finding in workers with vinyl chloride exposure.

\section{Case 3}

A 75 year old female with essential thrombocythaemia (JAK2 positive) managed with hydroxyurea presented with large volume ascites, leucocytosis and abnormal liver function tests.

Her initial ultrasound revealed splenomegaly and multiple low echogenicity lesions within the spleen. A subsequent abdominal computerised tomography scan showed normal enhancement of the liver with splenomegaly.

A bone marrow biopsy was performed and was consistent with post-essential thrombocythaemia myelofibrosis. Cytogenetic studies were normal. A transjugular liver biopsy to investigate deranged liver function tests showed minor extra-medullary haematopoiesis and diffuse fibrosis. No megakaryocytes were present on ascitic fluid cytology.

The patient's condition deteriorated over three weeks with the development of renal failure and diuretic refractory ascites. The patient subsequently died.

At autopsy the patient was found to have disseminated angiosarcoma involving the liver, spleen, omentum, peritoneum, peripancreatic and mesenteric lymph nodes. The portal vein was also invaded by angiosarcoma. Post mortem liver histology showed a classical pattern of primary angiosarcoma, with extensive but patchy involvement of the liver parenchyma. The antemortem liver biopsy was reviewed. No tumour was identified, and the negative biopsy was attributed to sampling error. 


\section{Discussion}

Our hospital is a tertiary referral metropolitan hospital with a liver transplantation unit. The three cases of hepatic angiosarcoma described in this series were seen over a 10 year period. All cases were diagnostically difficult.

Whilst hepatic angiosarcoma remains a very rare tumour it must be considered as a differential diagnosis in patients with rapidly deteriorating liver synthetic function with no clear aetiological factors. The first case was on the oral contraceptive pill which has been linked with multiple hepatic tumours but there is minimal evidence to suggest a clear association with hepatic angiosarcoma [4]. One of the cases was apparently sporadic, in the other the history of vinyl chloride exposure was only recognised when further questioning of the family was prompted by the autopsy findings. Our cases also illustrated diverse clinical presentations, with a Budd-Chiari like syndrome, hepatic failure supervening on a background of unusual chronic liver disease and malignant ascites.

The prognosis of hepatic angiosarcoma is dismal. Although treatment options remain limited, earlier diagnosis could enable resection which has been shown in a recent case series to have a 5 year survival of $40 \%$ [5].

Kim et al. [6] retrospectively analysed chemotherapy outcomes with selected regimens including doxorubicin, carboplatin, 5-fluorouracil, paclitaxel and bevacizumab for advanced hepatic angiosarcoma. Three patients died within 3 months of diagnosis, the remaining 2 received second or third line chemotherapy regimens and survived for 16 and 9 months, respectively.

As demonstrated in the above case series, the radiological findings of hepatic angiosarcoma are highly variable. They can range from single, or multiple lesions to diffuse infiltration of the liver. When compared with hepatocellular carcinoma they tend to have prolonged enhancement in the venous and delayed phases. However, as we have shown in case 3, the liver can also have a normal appearance on CT.

In conclusion, our series demonstrates that hepatic angiosarcoma remains a relevant differential diagnosis in current hepatology practice. Recognition requires a high index of suspicion for clinicians, radiologists and pathologists.

\section{Acknowledgement}

Liver transplant Unit, Austin Hospital, Victoria, Australia.

\section{References}

1. Mani H, Van Thiel DH (2001) Mesenchymal tumors of the liver. Clin Liver Dis 5: 219-57.

2. Zimmerman HJ, Lewis JH (1995) Chemical- and toxin-induced hepatotoxicity. Gastroenterol Clin North Am 24: 1027-45.

3. Locker GY, Doroshow JH, Zwelling LA, Chabner BA (1979) The clinical features of hepaticangiosarcoma: a report of four cases and a review of the English literature. Medicine 58: 48-64.

4. Shi EC, Fischer A, Crouch R, Ham JM (1981) Possible association of angiosarcoma with oral contraceptive agents. Med J Aust 1: $473-4$.

5. Duan FX, Li Q (2012) Primary hepatic angiosarcoma: A retrospective analysis of 6 cases, J Dig Dis 13: 381-5.

6. Kim HR, Rha SY, Cheon SH, Roh JK, Park YN, et al. (2009) Clinical features and treatment outcomes of advanced stage primary hepatic angiosarcoma. Ann Oncol 20: 780-7. 